\title{
Plasma inflammatory and vascular homeostasis biomarkers in newborns are not affected by mothers' oily fish intake
}

\author{
C. E. Garcia-Rodriguez ${ }^{1}$, M. D. Mesa ${ }^{1}$, J. Olza ${ }^{1}$, C. M. Aguilera ${ }^{1}$, E. A. Miles ${ }^{2}$, P. S. Noakes ${ }^{2}$, \\ M. Vlachava ${ }^{2}$, L. S. Kremmyda ${ }^{2}$ N. D. Diaper ${ }^{2}$, K. M. Godfrey ${ }^{2,3,4}$, P. C. Calder ${ }^{2,4}$ and A. Gil ${ }^{1}$ \\ ${ }^{1}$ Department of Biochemistry and Molecular Biology II, Institute of Nutrition and Food Technology "José Mataix", \\ Biomedical Research Center, University of Granada, Granada, Spain, ${ }^{2}$ Human Development and Health Academic Unit, \\ Faculty of Medicine, University of Southampton, Southampton, United Kingdom, ${ }^{3}$ Southampton Medical Research Council \\ Lifecourse Epidemiology Unit, University of Southampton, Southampton, United Kingdom and ${ }^{4}$ Southampton NIRH \\ Nutrition, Diet and Lifestyle Biomedical Research Unit, University Hospital Southampton NHS Foundation Trust, \\ Southampton, United Kingdom
}

The ingestion of (n-3) LC-PUFA may lower the concentrations of inflammatory biomarkers; therefore, we investigated whether the consumption of oily fish affects the levels of inflammatory cytokines and vascular adhesion factors in newborns.

Pregnant women $(n=123)$ were randomly assigned to continue their habitual diet (control group, $n=61)$, which was low in oily fish, or to consume two 150-g salmon portion per week (salmon group, $n=62$ ) (providing $3.45 \mathrm{~g}$ EPA plus DHA) from 20 week gestation until delivery. Salmon for use in the study was raised using dietary ingredients selected to contain low levels of contaminants Plasma inflammatory cytokines and vascular adhesion factors were measured in maternal samples at 38 weeks of gestation and cord blood samples, collected immediately after delivery. Differences between mothers and newborns were evaluated by Student t-tests. Correlations were estimated by computing Pearson's correlation coefficient in the case of normally distributed values and the Spearman correlation coefficient in the case of other distributions, respectively. $P$ values $<0.05$ were considered to be statistically significant. In the table, values are mean \pm SEM. Significant differences between mothers and their newborns are indicated in the table with $*$ and differences between groups with bold type. All statistical analyses were performed with the Statistical Package for Social Science 15.0 for Windows.

\begin{tabular}{|c|c|c|c|c|}
\hline & \multicolumn{4}{|c|}{ Group } \\
\hline & \multicolumn{2}{|c|}{ Control $(n=50)$} & \multicolumn{2}{|c|}{ Salmon $(n=51)$} \\
\hline & 38 weeks & Newborn & 38 weeks & Newborn \\
\hline IL-8 (ng/L) & $0.62 \pm 0.04$ & $7.45 \pm 2.72$ & $0.78 \pm 0.09$ & $2.74 \pm 0.54$ \\
\hline IL-6 (ng/L) & $1.04 \pm 0.14$ & $16.14 \pm 8.11$ & $1.84 \pm 0.57$ & $4.95 \pm 1.26$ \\
\hline TNF- $\alpha(n g / L)$ & $2.74 \pm 0.21$ & $16.92 \pm 9.18$ & $2.64 \pm 0.18$ & $6.77 \pm 0.32$ \\
\hline MCP-1 (ng/L) & $69 \pm 5$ & $222 \pm 24 *$ & $76 \pm 5$ & $253 \pm 36^{*}$ \\
\hline HGF (ng/L) & $907 \pm 101$ & $7186 \pm 492 *$ & $861 \pm 98$ & $6307 \pm 461 *$ \\
\hline NGF (ng/L) & $10.8 \pm 2.0$ & $12.0 \pm 1.4$ & $6.05 \pm 1.15$ & $11.21 \pm 2.31$ \\
\hline sE-selectin (vg/L) & $16.4 \pm 1.4$ & $77.3 \pm 5.7 *$ & $17.50 \pm 1.63$ & $82.68 \pm 6.13$ \\
\hline sICAM-1 (vg/L) & $176 \pm 28$ & $81 \pm 7^{*}$ & $121 \pm 5$ & $76 \pm 3^{*}$ \\
\hline sVCAM-1 (mg/L) & $1.00 \pm 0.05$ & $1.52 \pm 0.07 *$ & $1.03 \pm 0.05$ & $1.66 \pm 0.10^{*}$ \\
\hline tPAI-1(vg/L) & $38.8 \pm 2.3$ & $53.9 \pm 7.6$ & $36.6 \pm 3.2$ & $52.8 \pm 9.8$ \\
\hline MMP-9 (vg/L) & $101 \pm 11$ & $211 \pm 22 *$ & $71 \pm 8$ & $198 \pm 21 *$ \\
\hline
\end{tabular}

Significant correlations were observed between maternal and cord plasma for MCP-1, NGF and sICAM-1, only in the control group ( $P$ ranging between $<0.001$ and 0.030 ).

Therefore, inflammatory and vascular homeostasis biomarkers in newborns are not affected by the increased intake of farmed salmon. This study was supported by the European Union Commission under Framework 6: Sustainable Aquafeeds to Maximize the Health Benefits of Farmed Fish for Consumers (AQUAMAX; FOOD-CT-2006-16249). 